\title{
DISECCIÓN SUBMUCOSA ENDOSCÓPICA EN CÁNCER GÁSTRICO INCIPIENTE*
}

\author{
Drs. José Galindo R. ${ }^{1}$, Jorge Rodríguez G. ${ }^{2,3}$, Enrique Norero M. ${ }^{1,3}$, \\ Gloria Aguayo B. ${ }^{4}$, Martha Pruyas A. ${ }^{4}$, Eva Nilsen V. ${ }^{4}$, Cristián Martínez B. ${ }^{3}$, \\ Sergio Báez V. ${ }^{3}$, Alfonso Díaz F. ${ }^{3}$, Alfonso Calvo B. ${ }^{2,3,5}$
}

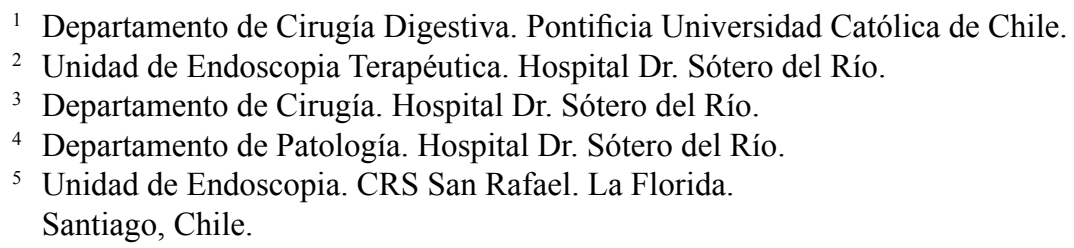

\begin{abstract}
\section{Endoscopic submucosal dissection for early gastric cancer}

Introduction: Endoscopic submucosal dissection (ESD) is nowadays the standard treatment for a subgroup of early gastric cancer with low risk of lymph node metastasis. This procedure has the advantage of achieving a higher percentage of negative margins and of allowing resections of larger tumors compared with the endoscopic mucosal resection (EMR) method, being less invasive compared with surgery. Aim: To analyze the postoperative outcomes, histology and overall survival of patients undergoing ESD in our center. Methods: Descriptive study. Data were collected from clinical records of patients undergoing ESD from January 2008 to June 2012. Results: 15 patients ( 8 males and 7 females, median age 70 years (45-88)) were included. The tumor was located at upper, middle and lower third in 2, 5 and 8 patients, respectively. Median tumor size was $13.5 \mathrm{~mm}$ (6-21). Most tumors were classified as type IIc. Among postoperative complications, there was 1 gastric bleeding and 3 gastric perforations. Hospital stay was 3 days (1-23). There was no mortality. There was one case of submucosal invasion. Negative margins were achieved in $86.7 \%$. There was one deep and one lateral positive margin, managed by surgery and re-ESD, respectively. At 16 months (7-61) of median follow-up, overall survival was $100 \%$. Tumor recurrence was observed in one patient at 51 months, was treated by surgery. Conclusion: ESD is a feasible technique in the minimally invasive management of early gastric cancer, allowing a high percentage of negative margins.
\end{abstract}

Key words: Early Gastric Cancer, endoscopic resection, endoscopy.

\section{Resumen}

Introducción: La disección endoscópica de la submucosa (DES) es actualmente el tratamiento estándar en pacientes con cáncer gástrico incipiente y bajo riesgo de metástasis ganglionares, con la ventaja de obtener

*Recibido el 13 de abril de 2015 y aceptado para publicación el 11 de mayo de 2015.

Los autores no refieren conflictos de interés.

Correspondencia: Dr. Alfonso Calvo B. acalvobelmar@yahoo.es 
un mayor porcentaje de márgenes negativos que en la mucosectomía y ser menos invasivo que la cirugía. Objetivo: Analizar los resultados inmediatos, anatomía patológica y sobrevida alejada de los pacientes sometidos a DES en nuestro centro. Métodos: Estudio descriptivo. Revisión de los registros clínicos de pacientes con lesiones gástricas incipientes sometidos a DES entre enero de 2008 y junio de 2012. Resultados: Serie compuesta por 15 pacientes, 8 de sexo masculino. Mediana de edad 70 años (45-88). Ubicación tumoral: tercio superior en 2, tercio medio en 5 y tercio inferior en 8. La mediana de tamaño tumoral fue 13,5 mm (6-21). La mayoría fueron lesiones IIc. Dentro de las complicaciones postoperatorias, hubo 1 hemorragia digestiva tratada por endoscopia y 3 perforaciones gástricas tratadas quirúrgicamente (gastrorrafia). Mediana de hospitalización, 3 días (1-23). No se registró mortalidad post-procedimiento. Se obtuvieron bordes negativos en un $86,7 \%$ de los casos. Un paciente presentó margen profundo positivo, tratado mediante gastrectomía subtotal y otro presentó positividad en un margen lateral, manejado con una nueva DES. Mediana de seguimiento 16 meses (7-61), sin fallecidos por patología tumoral. Hubo 1 recurrencia tumoral (51 meses), tratada quirúrgicamente. Conclusión: La DES representa una alternativa en el manejo de las lesiones gástricas incipientes, permitiendo un alto porcentaje de márgenes negativos.

Palabras clave: Cáncer gástrico incipiente, resección endoscópica, endoscopia.

\section{Introducción}

El cáncer gástrico representa la segunda causa de muerte por tumores malignos ${ }^{1}$. Su pronóstico sigue siendo sombrío, debido a que en la mayoría de los casos el diagnóstico es tardío. Basado en registros poblacionales, la sobrevida a cinco años en países occidentales no supera el $15 \%{ }^{2,3}$.

En 1962, la Sociedad Japonesa de Endoscopia Gastrointestinal definió el cáncer gástrico incipiente como aquél que infiltra la mucosa o submucosa, con o sin invasión ganglionar, siendo una enfermedad curativa si es detectada en esta etapa.

Tradicionalmente la cirugía radical ha sido el tratamiento de elección tanto para el cáncer gástrico incipiente como para el avanzado, con tasas de sobrevida a 5 años de $92,2 \%$ y $89,1 \%$ en cáncer mucoso y submucoso, respectivamente, y bajo porcentaje de recurrencia locorregional ${ }^{4,5}$. Estos pacientes tienen un riesgo de diseminación linfática de $3 \%$ para tumores mucosos y $18 \%$ para tumores submucosos ${ }^{6}$. Por lo tanto, existe un número importante de pacientes con cáncer gástrico incipiente que pueden ser tratados con terapias menos invasivas que la cirugía radical.

El primer caso de resección endoscópica en una lesión polipoídea maligna usando un asa de polipectomía fue publicado en 1974. En la década de los 80 , fueron publicadas varias técnicas de resección endoscópica de la mucosa (REM) usando diferentes tipos de accesorios. En la década de los 90 fueron definidas las características de las lesiones susceptibles de ser resecadas por vía endoscópica ${ }^{7,8}$. Sin embargo, la REM tiene varias desventajas, como la obtención de la pieza en parcialidades, aumento en la tasa de recurrencia local, dificultad para el análisis histológico y que sólo permite resecciones de tumores pequeños.
Actualmente, la disección endoscópica de la submucosa (DES) es la técnica de elección para el tratamiento del cáncer gástrico incipiente y ha permitido expandir los criterios para resecciones endoscópicas ${ }^{9-12}$.

Este estudio tiene por objetivo describir los resultados a corto plazo, el análisis histológico y la sobrevida global y libre de enfermedad de pacientes sometidos a DES para tumores gástricos incipientes en nuestro centro.

\section{Material y Método}

\section{Diseño}

Estudio retrospectivo. Los datos se obtuvieron por medio de una revisión de todos los procedimientos endoscópicos realizados en el Hospital Dr. Sótero del Río. Se incluyeron consecutivamente todos los pacientes con tumores gástricos incipientes tratados con DES entre enero de 2008 y junio de 2012.

La mayoría de los candidatos para DES fueron seleccionados a través del programa de detección de cáncer gástrico incipiente en la comuna de La Florida, Santiago ${ }^{13}$. Las características principales de los tumores gástricos (tamaño, localización, tipo, etc.) fueron revisadas de acuerdo a la clasificación Japonesa para cáncer gástrico incipiente ${ }^{14}$. Todas las DES fueron realizadas de acuerdo con los criterios extendidos de resección submucosa propuestos por Gotoda et $\mathrm{al}^{11}$.

\section{Técnica}

Los procedimientos fueron realizados en la Unidad de Endoscopia, previa obtención del consentimiento informado. Los procedimientos fueron llevados a cabo bajo sedación con propofol y monitorización cardiopulmonar. La evaluación preopera- 
toria incluyó un electrocardiograma y exámenes de laboratorio. El estudio de diseminación fue realizado por medio de ecografía abdominal o tomografía computada.

En todos los casos se utilizó un videoendoscopio Olympus Exera II $180^{\mathrm{TM}}$ de un canal. Se identifica el tumor y se delimitan los bordes utilizando Índigo Carmín o mediante Imágenes de Banda Angosta (Narrow Band Imaging, NBI). Los márgenes tumorales se marcan con puntos de coagulación aproximadamente $5 \mathrm{~mm}$ por fuera de la lesión. La submucosa se infiltra con solución salina más adrenalina o hidroxietil almidón en los casos más recientes. Luego se realiza una incisión circunferencial en la mucosa por fuera de los puntos de coagulación y posteriormente disección de la submucosa separándola del plano muscular, utilizando de preferencia IT Knife circular o triangular. Luego de resecada la pieza, se realiza hemostasia y se verifica la indemnidad de la pared gástrica. En las Figuras 1 y 2 se muestra un esquema del procedimiento e imágenes endoscópicas de una DES en un paciente de la serie.

La pieza resecada se extiende sobre una superficie y sus bordes son fijados con alfileres para evitar la retracción y facilitar el análisis histopatológico. Los hallazgos endoscópicos y los resultados de las biopsias fueron analizados en reunión conjunta con

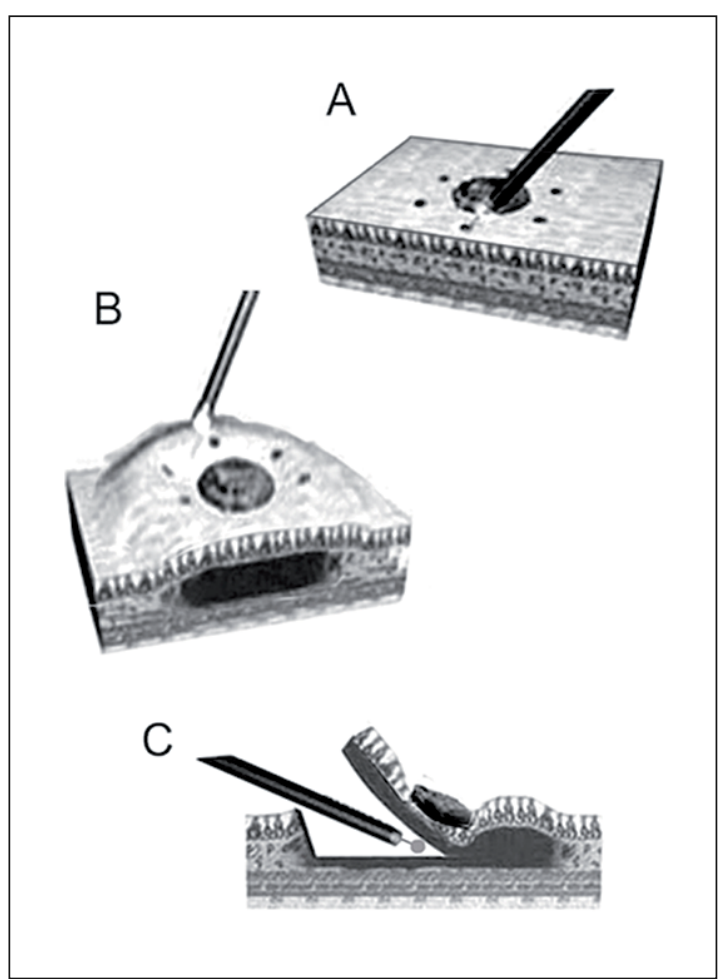

Figura 1. Esquematización de la DES. A) Marcación de los bordes; B) Infiltración de la submucosa; C) Disección.

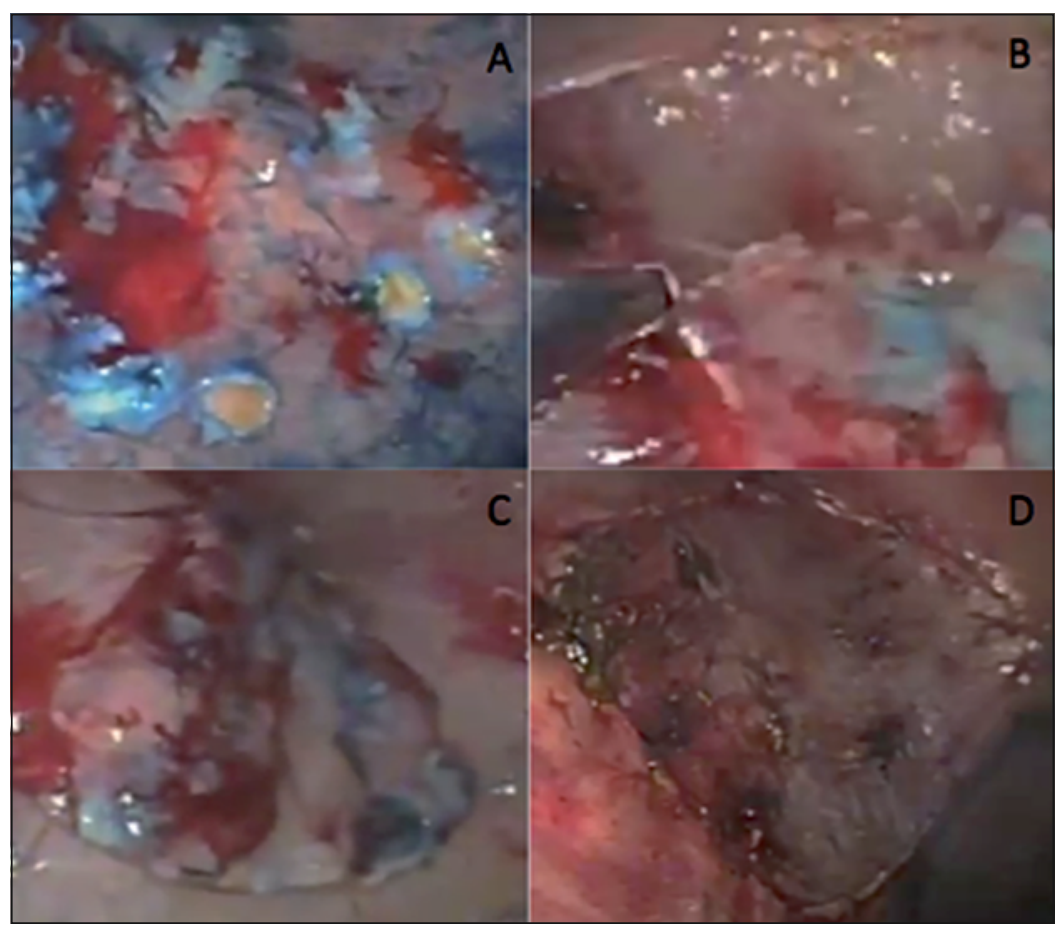

Figura 2. Imágenes endoscópicas de una DES realizada en un paciente de la serie. A) Marcación de los bordes; B) Disección utilizando IT Knife; C) Pieza completamente resecada; D) Lecho operatorio. 
al menos dos patólogos. El reporte histológico de la pieza resecada incluyó el diámetro de la lesión, la clasificación macroscópica, el tipo histológico con grado de diferenciación, el estado de los bordes lateral y profundo y la invasión vascular o linfática. Se consideró una resección curativa según los criterios propuestos por la sociedad Japonesa de cáncer gástrico ${ }^{14}$.

Una vez finalizado el procedimiento, los pacientes son llevados a recuperación breve y luego a sala común. Todos los pacientes reciben omeprazol y sucralfato desde el postoperatorio inmediato hasta 6-8 semanas. La alimentación comienza a las 48 o $72 \mathrm{~h}$.

\section{Análisis estadístico}

Se analizaron variables biodemográficas, características clínicas, técnica quirúrgica, morbilidad asociada al procedimiento, resultado histológico de la pieza operatoria y seguimiento. Los datos incluyeron edad, sexo, clasificación ASA ${ }^{15}$, características tumorales (localización, clasificación, tamaño), histología pre y postoperatoria, complicaciones intraoperatorias, morbilidad postoperatoria, estadía hospitalaria, mortalidad postoperatoria, recurrencia tumoral, sobrevida global y sobrevida libre de enfermedad.

Las variables categóricas se expresan en valores numéricos y porcentajes. Las variables continuas se expresan en medianas y rangos. El análisis estadís- tico re realizó con los programas SPSS ver. $2.0 \mathrm{y}$ GraphPadPrism ver. 5.0.

\section{Seguimiento}

El seguimiento se realizó en base a controles ambulatorios con endoscopia y biopsia rutinaria de la cicatriz a los 3, 6 y 12 meses, y luego anualmente.

\section{Aspectos éticos}

El estudio, análisis de los registros clínicos, endoscópicos y de anatomía patológica contó con la aprobación del Comité de Ética del hospital.

\section{Resultados}

Durante el período de estudio se realizaron 15 DES en 15 pacientes consecutivos con lesiones gástricas incipientes que cumplían los criterios. $\mathrm{La}$ mediana de edad fue de 70 años (extremos: 45-88). Ocho pacientes eran de sexo masculino $(53,3 \%)$. Un 73,3\% de los pacientes fueron clasificados como ASA II (Tabla 1).

De acuerdo con la clasificación japonesa para cáncer gástrico incipiente, todas las lesiones fueron clasificadas como tipo II. En 4 casos correspondieron a lesiones tipo IIa, en 3 casos a lesiones tipo IIb y en 10 casos a lesiones tipo IIc (Tabla 1).

Utilizando la división anatómica del estómago, las lesiones se ubicaron en el tercio superior en 2

Tabla 1. Características preoperatorias de los pacientes sometidos a DES

\begin{tabular}{|ccccccc|}
\hline $\mathbf{n}$ & Sexo & Edad & ASA score & Clasificación & Localización tumoral & Localización tumoral (circunferencial) \\
\hline 1 & F & 70 & II & II b + II c & S & Cme \\
2 & M & 79 & II & II c & M & Cma \\
\hline 3 & F & 53 & II & II c & S & Post \\
\hline 4 & M & 67 & I & II c & I & Cma \\
\hline 5 & F & 64 & II & II b & I & Ant \\
\hline 6 & M & 88 & II & II c & I & Cma \\
\hline 7 & F & 45 & I & II a & I & Cme \\
\hline 8 & M & 85 & II & II a + II c & I & Cme \\
\hline 9 & F & 64 & II & II c & I & Ant \\
\hline 10 & F & 80 & II & II a & I & Cme \\
\hline 11 & M & 56 & II & II b & M & Cma \\
\hline 12 & F & 85 & I & II a & M & Cme \\
\hline 13 & M & 75 & II & II c & M & Cme \\
\hline 14 & M & 56 & II & II c & M & Cme \\
\hline 15 & M & 75 & I & II c & I & Ant: Catant \\
\hline
\end{tabular}

S: Tercio Superior; M: Tercio Medio; I: Tercio Inferior; Cma: Curvatura Mayor; Cme: Curvatura Menor; Ant: Cara Anterior; Post: Cara Posterior. 
Tabla 2. Análisis histológico de las piezas obtenidas por DES

\begin{tabular}{|cccccc|}
\hline n & $\begin{array}{c}\text { Tamaño tumoral endoscopia } \\
\text { preoperatoria (mm) }\end{array}$ & $\begin{array}{c}\text { Histología } \\
\text { preoperatoria }\end{array}$ & $\begin{array}{c}\text { Histología } \\
\text { final }\end{array}$ & $\begin{array}{c}\text { Tamaño tumoral DES } \\
\text { (mm) }\end{array}$ & $\begin{array}{c}\text { Margen } \\
\text { positivo }\end{array}$ \\
\hline 1 & 6 & AC & AC & 9 & - \\
\hline 2 & 7 & AC & AC & ND & - \\
\hline 3 & 15 & AC & AC & 21 & - \\
\hline 4 & ND & AC & AC & 18 & Vertical \\
\hline 5 & 10 & AC & DAG & 10 & - \\
\hline 6 & 6 & AC & AC & 20 & - \\
\hline 7 & 5 & DAG & DAG & ND & - \\
\hline 8 & 16 & AC & DAG & 6 & - \\
\hline 9 & 12 & AC & AC & 12 & - \\
\hline 10 & 10 & DAG & AC & ND & - \\
\hline 11 & 15 & AC & AC & 18 & Lateral \\
\hline 12 & 15 & DAG & AC & 15 & - \\
\hline 13 & 15 & AC & AC & 15 & - \\
\hline 14 & 12 & AC & AC & 12 & - \\
\hline 15 & 5 & AC & AC & 6 & \\
\hline
\end{tabular}

AC: Adenocarcinoma; DAG: Displasia de alto grado; ND: Sin dato.

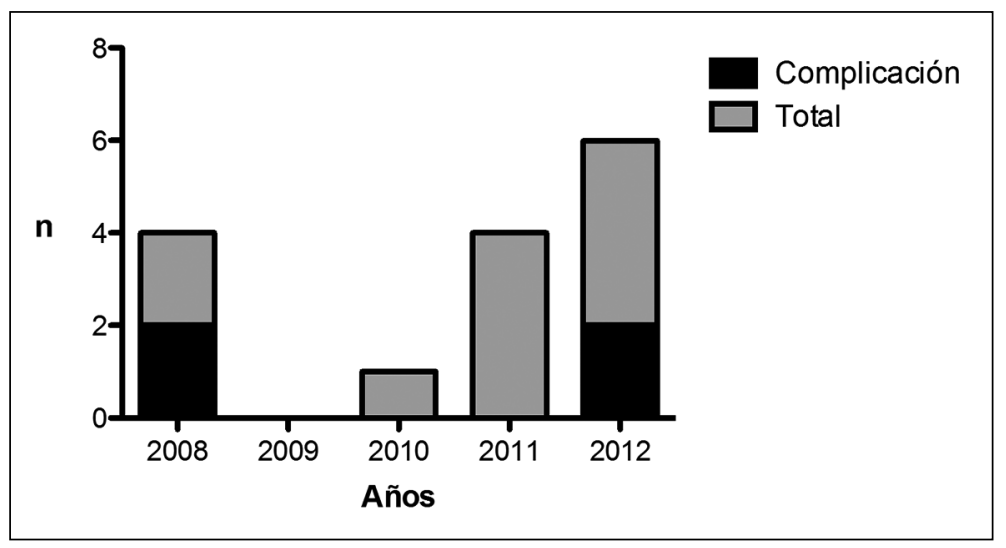

Figura 3. Curva de morbilidad de los pacientes sometidos a DES, por año.

pacientes, en el tercio medio en 5 pacientes y en el tercio inferior en 8 pacientes. En relación a la circunferencia gástrica, la mayoría de las lesiones se ubicó en la curvatura menor ( 8 pacientes), en 4 casos se ubicó en la curvatura mayor, en 2 casos en la cara anterior y en 1 caso en la cara posterior (Tabla 1).

La biopsia preoperatoria fue compatible con adenocarcinoma en 12 pacientes y con displasia de alto grado (DAG) en 3, los cuales fueron sometidos a DES debido al aspecto endoscópico de las lesiones, altamente sugerente de malignidad, luego de repetidas biopsias con el diagnóstico de DAG (Tabla 2).
Con respecto a las complicaciones relacionadas al procedimiento, se diagnosticaron tres perforaciones gástricas, dos de las cuales corresponden a los primeros casos de la serie, que fueron tratadas quirúrgicamente mediante gastrorrafia. Por otra parte, hubo una hemorragia digestiva del sitio de disección, que fue controlada endoscópicamente por medio de clips. La curva de morbilidad se describe en la Figura 3. La mediana de estadía hospitalaria fue de 3 días (extremos: 1-23). No hubo mortalidad asociada al procedimiento.

El análisis histológico definitivo de las piezas 


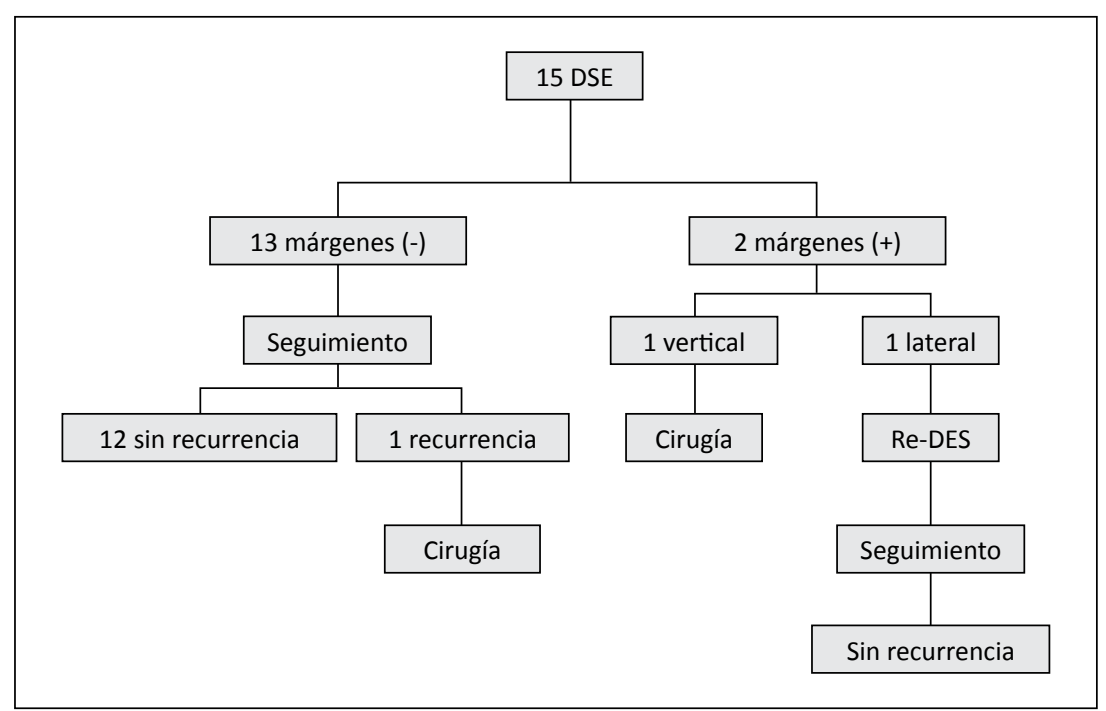

Figura 4. Resultados oncológicos de los pacientes sometidos a DES. resecadas mostró adenocarcinoma en 12 pacientes y DAG en tres. En dos de los pacientes con diagnóstico preoperatorio de DAG $(66,7 \%)$ se encontró adenocarcinoma en la pieza resecada. Según el grado de diferenciación, en 3 casos el tumor fue catalogado como bien diferenciado, en 8 casos como moderadamente diferenciado y en 1 caso fue descrito como poco diferenciado (lesión de $7 \mathrm{~mm}$ ). La mediana del tamaño de la lesión fue de 13,5 mm (extremos: 6-21). En ningún paciente se describió permeaciones linfáticas, vasculares o perineurales. En un paciente $(8,3 \%)$ se informó invasión de la submucosa. Dos pacientes presentaron bordes positivos $(16,6 \%)$ (Tabla 2). En el primer caso se trató del borde profundo (infiltración de la submucosa), que fue tratado mediante gastrectomía subtotal laparoscópica, en cuya biopsia no se encontraron elementos de malignidad. El segundo caso corresponde a un paciente con borde lateral positivo, que fue tratado mediante una nueva resección endoscópica. Los controles posteriores no han mostrado recidiva tumoral (Figura 4). En total, en un $85,7 \%$ la resección fue considerada como curativa.

Se realizó seguimiento a todos los pacientes, con una mediana de 16 meses (extremos: 7-61). Durante este período se registró sólo una recidiva tumoral, diagnosticada a los 51 meses desde la resección. El paciente rechazó una nueva resección endoscópica y fue tratado con una gastrectomía subtotal realizada mediante cirugía clásica, con buena evolución postoperatoria y controles oncológicos sin evidencia de una nueva recidiva. No hubo pacientes fallecidos por patología tumoral durante el seguimiento, al término de este estudio.

\section{Discusión}

La cirugía radical ha sido el tratamiento de elección en cáncer gástrico incipiente, primero por laparotomía y en los últimos años mediante el uso de laparoscopia. Sin embargo, no está exenta de morbimortalidad y complicaciones alejadas, como los síndromes post-gastrectomía, que afectan la calidad de vida de los pacientes. La resección endoscópica presenta la ventaja de preservar completamente el estómago y además reduce la morbilidad, el tiempo operatorio, la estadía hospitalaria y los costos relacionados $^{16}$.

La evaluación preoperatoria de los pacientes con cáncer gástrico es un ítem importante para determinar la correcta modalidad de tratamiento. En este sentido, la endosonografía se ha convertido en una herramienta de utilidad para la evaluación de la profundidad tumoral y el compromiso ganglionar ${ }^{17}$. Sin embargo, es un procedimiento operador dependiente, que incrementa los costos, y que no está exento de morbilidad. En un meta-análisis reciente, se concluye que su eficacia diagnóstica para la profundidad en la pared es de $75 \%$ y para el compromiso ganglionar de $64 \%$, siendo más certera en la evaluación de tumores avanzados (T3 y T4) ${ }^{18}$. En la serie presentada no se utilizó la endosonografía como herramienta complementaria al diagnóstico endoscópico, principalmente por la no disponibilidad del recurso en nuestro centro.

La primera técnica endoscópica resectiva utilizada para lesiones incipientes fue la REM; sin embargo, está limitada técnicamente a lesiones bien diferenciadas, diámetro menor o igual a $20 \mathrm{~mm}$, sin 
ulceración ni cicatrices. Aun cuando es una técnica relativamente simple y con baja tasa de complicaciones, presenta un mayor riesgo de recurrencia local debido al porcentaje de resecciones incompletas, con márgenes comprometidos o que no pueden ser evaluados adecuadamente en la histología, en su mayoría debido a resecciones en dos o más fragmentos.

Basado en un número significativo de pacientes operados por cáncer gástrico incipiente se ha podido establecer que existe un subgrupo de éstos que presentan un mínimo riesgo de compromiso ganglionar, lo que ha permitido expandir los criterios de resección endoscópica a adenocarcinomas bien o moderadamente diferenciados, sin limitación de tamaño si son intramucosos y sin ulceración, o menores de $30 \mathrm{~mm}$ si presentan ulceración, infiltración submucosa menor de $500 \mu \mathrm{m}$ (SM1), y cánceres indiferenciados menores de $20 \mathrm{~mm}$, sin ulceración ${ }^{11}$. La DES se ha convertido en el tratamiento estándar en este subgrupo de pacientes. En comparación con la REM, esta técnica permite realizar resecciones en lesiones de mayor tamaño, mayoritariamente en un fragmento, disminuyen las recurrencias locales y permite un adecuado estudio histológico, con el cual se logra establecer si el procedimiento fue curativo o si se requiere terapia adicional ${ }^{19-21}$.

Esto se ha traducido en que durante los últimos años la DES ha reemplazado a la cirugía radical para el tratamiento del cáncer gástrico incipiente en países con altas tasas de cáncer, como Japón y Corea $^{22,23}$. En países occidentales lentamente se ha adoptado esta técnica, incrementándose el número de pacientes cada año ${ }^{24-26}$. La DES es un procedimiento técnicamente más complejo que la REM, requiere mayor habilidad y experiencia por parte del endoscopista, y requiere un mayor número de accesorios. Las complicaciones son fundamentalmente la perforación de la pared gástrica y la hemorragia. Comparativamente, la DES presenta una mayor tasa de perforación que la REM, pero la hemorragia es similar en ambas técnicas ${ }^{20}$.

En nuestra serie, los tres casos asociados a perforación fueron tratados mediante laparotomía y sutura de la perforación, sin morbilidad ni mortalidad asociada al procedimiento. Este porcentaje mayor a las series orientales podría ser explicado en parte por la curva de aprendizaje en la técnica quirúrgica, ya que dos de los tres casos $(66,7 \%)$ corresponden al primer y segundo caso de la serie.

En nuestra experiencia se observó un episodio de hemorragia digestiva, que ocurrió en el intraoperatorio y fue manejado exitosamente por vía endoscópica.

La interpretación de la histología en tumores incipientes es difícil, tanto en la biopsia endoscópica como en la biopsia de resección. Además del cono- cimiento y experiencia requeridos para la interpretación adecuada de estas lesiones, existen diferencias de criterio entre patólogos japoneses y occidentales en cuanto a la definición de cáncer. Por otra parte, puede haber variaciones interobservador entre patólogos con similar formación ${ }^{27}$. Con el objetivo de evitar estas discrepancias se llegó a un consenso entre patólogos japoneses y occidentales, denominado "Clasificación de Viena de Neoplasias Epiteliales Gastrointestinales", que agrupa las lesiones en 5 categorías y que permite a los clínicos decidir la conducta de acuerdo al resultado de la biopsia ${ }^{28}$. En esta serie y de acuerdo al protocolo se han resecado aquellos pacientes de la categoría 4 y 5 . De los 15 pacientes, hubo correlación positiva entre la biopsia endoscópica y quirúrgica en 11. En 2 pacientes con DAG la biopsia definitiva mostró cáncer y en 2 con diagnóstico de cáncer el diagnóstico definitivo fue DAG. Finalmente, un paciente presentó una lesión con varias biopsias con diagnóstico de DAG; fue considerada una lesión neoplásica de acuerdo con la clasificación de Viena (carcinoma intramucoso de acuerdo al criterio japonés) y fue resecada.

En grandes series el porcentaje de resecciones en un fragmento es aproximadamente $95 \%$, con un porcentaje de márgenes negativos entre $66 \%$ y $88 \%$. En nuestra serie, en 2 de los 14 pacientes con diagnóstico final de adenocarcinoma gástrico se encontró margen positivo, por lo que el porcentaje de resecciones curativas fue de $85,7 \%$. Los dos pacientes con resecciones no curativas fueron sometidos a tratamiento complementario (re-DES para margen lateral y cirugía para margen profundo). Esto apunta a la gran importancia del análisis histopatológico detallado en la evaluación de la curabilidad de la resección.

El seguimiento de estos pacientes se realiza por medio de endoscopia con biopsia de la zona cicatricial aun cuando no exista sospecha de recidiva. Esto permite un diagnóstico precoz de una recurrencia, de un cáncer sincrónico no advertido en el estudio preoperatorio y la aparición de un cáncer metacrónico, situación que se presentó en un paciente de nuestra serie. Esto apoya el seguimiento continuo y a largo plazo, que debe incluir además la erradicación de Helicobacter pylori, tratamiento que de acuerdo con la evidencia disminuye el riesgo de cáncer metacrónico ${ }^{29}$.

De acuerdo a nuestro conocimiento, este estudio es el primer reporte nacional de DES en el tratamiento de lesiones gástricas incipientes. De esta breve experiencia se puede concluir que se trata de un procedimiento factible de realizar en nuestro medio. Esta es una técnica validada con miles de casos tratados en Japón y Corea con ventajas importantes sobre la cirugía en un subgrupo de tumores incipien- 
tes. Dada la alta tasa de cáncer gástrico en nuestro país, uno de los desafíos es la detección precoz, que permitiría aumentar el número de procedimientos endoscópicos en diferentes centros, mejorando la sobrevida para esta enfermedad.

\section{Agradecimientos}

Agradecemos a las técnicos María Alicia Correa, Claudia Velásquez y Patricia Verdugo, por su participación en la evaluación preoperatoria de los pacientes, asistencia durante los procedimientos y seguimiento.

\section{Referencias}

1. Ferlay J, Shin HR, Bray F, Forman D, Mathers C, Parkin DM. Estimates of worldwide burden of cancer in 2008: GLOBOCAN 2008. Int J Cancer 2010;127:2893-917.

2. Heise K, Bertran E, Andia ME, Ferreccio C. Incidence and survival of stomach cancer in a high-risk population of Chile. World J Gastroenterol. 2009;15:1854-62.

3. Coupland VH, Allum W, Blazeby JM, Mendall MA, Hardwick RH, Linklater KM, et al. Incidence and survival of oesophageal and gastric cancer in England between 1998 and 2007, a population-based study. BMC Cancer 2012;12:11. doi: 10.1186/1471-2407-12-11.

4. Isobe Y, Nashimoto A, Akazawa K, Oda I, Hayashi K, Miyashiro I, et al. Gastric cancer treatment in Japan: 2008 annual report of the JGCA nationwide registry. Gastric Cancer 2011;14:301-16.

5. Saka M, Katai H, Fukagawa T, Nijjar R, Sano T. Recurrence in early gastric cancer with lymph node metastasis. Gastric Cancer 2008;11:214-8.

6. Gotoda T, Yanagisawa A, Sasako M, Ono H, Nakanishi Y, Shimoda T, et al. Incidence of lymph node metastasis from early gastric cancer: estimation with a large number of cases at two large centers. Gastric Cancer 2000;3:219-25

7. Shim CS. Endoscopic mucosal resection: an overview of the value of different techniques. Endoscopy 2001;33:271-5.

8. Ono H, Kondo H, Gotoda T, Shirao K, Yamaguchi $\mathrm{H}$, Saito D, et al. Endoscopic mucosal resection for treatment of early gastric cancer. Gut 2001;48:225-9.

9. Oda I, Gotoda T, Hamanaka H, Eguchi T, Saito Y, Matsuda T, et al. Endoscopic submucosal dissection for early gastric cancer: technical feasibility, operation time and complications from a large consecutive series. Digestive Endoscopy 2005;17:54-8.

10. Kakushima N, Fujishiro M. Endoscopic submucosal dissection for gastrointestinal neoplasms. World J Gastroenterol. 2008;14:2962-7.

11. Gotoda T, Yamamoto H, Soetikno RM. Endoscopic submucosal dissection of early gastric cancer. J Gastroenterol. 2006;41:929-42.

12. Tanaka M, Ono H, Hasuike N, Takizawa K. Endoscopic submucosal dissection of early gastric cancer. Digestion 2008;77 Suppl 1:23-8.

13. Calvo BA, Pruyas AM, Nilsen VE, Verdugo LP. Pesquisa poblacional de cáncer gástrico en pacientes sintomáticos digestivos, período 1996-2000. Rev Med Chile 2001;129:749-55.

14. Japanese classification of gastric carcinoma: 3rd English edition. Gastric Cancer. 2011;14:101-12.

15. Daabiss M. American Society of Anaesthesiologists physical status classification. Indian $\mathrm{J}$ Anaesth. 2011;55:111-5.

16. Chiu PW, Teoh AY, To KF, Wong SK, Liu SY, Lam CC, et al. Endoscopic submucosal dissection (ESD) compared with gastrectomy for treatment of early gastric neoplasia: a retrospective cohort study. Surg Endosc. 2012;26:3584-91.

17. Polkowski M. Endosonographic staging of upper intestinalmalignancy. Best Pract Res Clin Gastroenterol. 2009;23:649-61.

18. Cardoso R, Coburn N, Seevaratnam R, Sutradhar R, Lourenco LG, Mahar A, et al. A systematic review and meta-analysis of the utility of EUS for preoperative staging for gastric cancer.Gastric Cancer 2012;15 Suppl 1:S19-26.

19. Oda I, Saito D, Tada M, Iishi H, Tanabe S, Oyama T, et al. A multicenter retrospective study of endoscopic resection for early gastric cancer. Gastric Cancer 2006;9:262-70.

20. Lian J, Chen S, Zhang Y, Qiu F. A meta-analysis of endoscopic submucosal dissection and EMR for early gastric cancer. Gastrointest Endosc. 2012;76:763-70.

21. Oka S, Tanaka S, Kaneko I, Mouri R, Hirata M, Kawamura $\mathrm{T}$, et al. Advantage of endoscopic submucosal dissection compared with EMR for early gastric cancer. Gastrointest Endosc. 2006;64:877-83.

22. Ohta T, Ishihara R, Uedo N, Takeuchi Y, Nagai K, Matsui $\mathrm{F}$, et al. Factors predicting perforation during endoscopic submucosal dissection for gastric cancer. Gastrointest Endosc. 2012;75:1159-65.

23. Chung IK, Lee JH, Lee SH, Kim SJ, Cho JY, Cho WY, et al. Therapeutic outcomes in 1000 cases of endoscopic submucosal dissection for early gastric neoplasms: Korean ESD Study Group multicenter study. Gastrointest Endosc. 2009;69:1228-35.

24. Cardoso DM, Campoli PM, Yokoi C, Ejima FH, Barreto PA, de Brito AM, et al. Initial experience in Brazil with endoscopic submucosal dissection for early gastric cancer using insulation-tipped knife: a safety and feasibility study. Gastric Cancer 2008;11:226-32.

25. Dinis-Ribeiro M, Pimentel-Nunes P, Afonso M, Costa N, Lopes C, Moreira-Dias L. A European case series of endoscopic submucosal dissection for gastric superficial lesions. Gastrointest Endosc. 2009;69:350-5. 
26. Probst A, Pommer B, Golger D, Anthuber M, Arnholdt $\mathrm{H}$, Messmann $\mathrm{H}$. Endoscopic submucosal dissection in gastric neoplasia-experience from a European center. Endoscopy 2010;42:1037-44.

27. Schlemper RJ, Itabashi M, Kato Y, Lewin KJ, Riddell $\mathrm{RH}$, Shimoda T, et al. Differences in diagnostic criteria for gastric carcinoma between Japanese and western pathologists. Lancet 1997;349(9067):1725-9.

28. Schlemper RJ, Riddell RH, Kato Y, Borchard F, Coo- per HS, Dawsey SM, et al. The Vienna classification of gastrointestinal epithelial neoplasia. Gut 2000;47:2515.

29. Fukase K, Kato M, Kikuchi S, Inoue K, Uemura N, Okamoto S, et al. Effect of eradication of Helicobacter pylori on incidence of metachronous gastric carcinoma after endoscopic resection of early gastric cancer: an open-label, randomised controlled trial. Lancet 2008;372(9636):392-7. 